\title{
SEMI-ISOTOPIES AND THE LATTICE OF INNER IDEALS OF CERTAIN QUADRATIC JORDAN ALGEBRAS
}

\author{
BY
}

\author{
JEROME M. KATZ(1)
}

\begin{abstract}
The concept of isotopy plays an extremely important role in the structure theory of simple quadratic Jordan algebras satisfying the minimum condition on principal inner ideals. We take Koecher's characterization of isotopy and use it as the basis of a definition of semi-isotopy.

It is clear that semi-isotopies induce, in a natural way, automorphisms of the lattice of inner ideals. We concern ourselves with the converse problem; namely, if $\eta$ is a semilinear bijection of a quadratic Jordan algebra such that $\eta$ induces an automorphism of the lattice of inner ideals, is $\eta$ necessarily a semiisotopy? We answer the above question in the affirmative for a large class of simple quadratic Jordan algebras satisfying the minimum condition on principal inner ideals (said class includes all such algebras of capacity at least three over fields of characteristic unequal to two). Moreover, we prove that the only such maps which induce the identity automorphism on the lattice are the scalar multiplications.
\end{abstract}

1. Preliminaries. In this paper, we continue our study of automorphisms of the lattice of inner ideals of simple quadratic Jordan algebras begun in our earlier paper [7]. In that paper, attention was focused on determining the automorphism group of the lattice of inner ideals and on determining under what conditions two algebras can have isomorphic lattices of inner ideals. In the present paper our attention is focused on determining which semilinear bijections of a simple quadratic Jordan algebra satisfying the minimum condition on principal inner ideals induce automorphisms of the lattice of inner ideals. We will freely make use of our earlier results and of McCrimmon's determination of the inner ideals for the algebras under consideration [9].

The concept of an isotope of a quadratic Jordan algebra plays an extremely important role in the structure theory (see [3]). One defines an isotopy to be an isomorphism of a quadratic Jordan algebra onto an isotope.

Received by the editors July $20,1973$.

AMS (MOS) subject classifications (1970). Primary 17C20, 17C30; Secondary 16A68.

Key words and phrases. Quadratic Jordan algebra, inner ideal, semi-isotopy, structure group.

(1) Research supported in part by a University of Missouri-St. Louis summer faculty fellowship.

Copyright $\odot 1974$, American Mathematical Society 
It is well known [3, p. 1.67] that a bijective linear mapping $\eta$ of a quadratic Jordan algebra $\mathcal{Y}$ onto itself is an isotopy if and only if there exists a linear mapping $\eta^{*}$ of $\Im$ such that

$$
U_{a \eta}=\eta^{*} U_{a} \eta \text { for all } a \in \Im .
$$

We define a bijective semilinear mapping $\eta$ to be a semi-isotopy if there exists a semilinear mapping $\eta^{*}$ of $\}$ such that (1) holds. The set of all semi-isotopies of $\mathcal{Y}$ forms a group.

It is easily seen that the image of an inner ideal under a semi-isotopy is an inner ideal. Thus every semi-isotopy induces in a natural way an automorphism of the lattice of inner ideals. It is our intention to show that if $\eta$ is a semilinear bijection of a quadratic Jordan algebra which induces an automorphism of the lattice of inner ideals then, under certain assumptions on the algebra, $\eta$ is a semiisotopy. The method of proof will be by a case-by-case examination of the simple quadratic Jordan algebras satisfying the minimum condition on principal inner ideals. We will make use of the classification of such algebras due to Jacobson, Osborn and McCrimmon (see [3] or [8]).

First, we prove the following general proposition concerning regular quadratic Jordan algebras (i.e. those in which $x \in \Im_{U_{x}}$ ).

Proposition 1. Suppose $\eta$ is a semilinear bijection of a regular quadratic Jordan algebra $\Im$ which induces an automorphism of the lattice of inner ideals of $\Im$. Then $\mathfrak{S}=\Im U_{c}$ if and only if $\mathfrak{S} \eta=\Im U_{c \eta}$.

Proof. Since $\eta^{-1}$ also induces such an automorphism, it suffices to prove that $\mathfrak{C}=\Im_{U_{c}}$ implies $\mathfrak{S} \eta=\Im_{c \eta}$.

If $c \in\left(\mathcal{S}, c \eta \in\left(S_{\eta}\right.\right.$ which implies $c \eta \in\left(\Im U_{c}\right) \eta$. Thus $\Im_{c \eta} \subseteq\left(\Im U_{c}\right) \eta$ since $\left(\Im U_{c}\right) \eta$ is an inner ideal.

Since $c \eta \in \Im U_{c \eta}, c=c \eta \eta^{-1} \in\left(\Im U_{c \eta}\right) \eta^{-1}$. But since $\left(\Im U_{c \eta}\right) \eta^{-1}$ is an inner ideal containing $c$, we must have

$$
\Im U_{c} \subseteq\left(\Im U_{c \eta}\right) \eta^{-1} \text { or }\left(\Im U_{c}\right) \eta \subseteq \Im_{c \eta},
$$

giving the desired equality.

We will be applying the conclusion of Proposition 1 to simple quadratic Jordan algebras satisfying the minimum condition on principal inner ideals, as these algebras are known to be regular.

2. The algebras Jord $(Q, 1)$. In this section we deal with quadratic Jordan algebras of quadratic forms with base points. In our previous paper [7] using results due to Dieudonné(see [1]), we showed that any automorphism of the lattice 
of inner ideals of a simple finite-dimensional algebra $\operatorname{Jord}(Q, 1)$, where $Q$ is a nondegenerate quadratic form of Witt index at least three, is induced by a semisimilarity of the form $Q$. The reader is referred to our previous paper [7] for a brief summary of terminology used in connection with these algebras. Jacobson and McCrimmon [5, Theorem 8] have essentially shown that semisimilarities of the form $Q$ coincide with semi-isotopies of the algebra Jord $(Q, 1)$. Thus to prove that any semilinear bijection of Jord $(Q, 1)$ that induces an automorphism of the lattice of inner ideals is a semi-isotopy, it suffices to show that any semilinear bijection that induces the identity automorphism on the lattice of inner ideals is a semi-isotopy. Thus we prove the following lemma.

LEMMA 1. Let $\Im=$ Jord $(Q, 1)$ where $(Q, 1)$ is a nondegenerate quadratic form of Witt index at least three and $\mathcal{Y}$ is finite dimensional. Suppose $\eta$ is a semilinear bijection of $\mathfrak{\Im}$ such that $\mathfrak{S} \eta=\mathfrak{S}$ for every inner ideal $\mathfrak{S}$ of $\mathfrak{\Im}$. Then $\eta$ is a scalar multiplication.

Proof. We recall [9, Theorem 6] that the proper inner ideals of $\Im$ are precisely the totally singular subspaces of $\Im$.

Let $v_{1}, v_{2}, \cdots, v_{k}$ be a basis for a maximal totally singular subspace of $\Im$ and $v_{1}^{*}, v_{2}^{*}, \cdots, v_{k}^{*}$ be a dual basis to it. Then there exist $\alpha_{i}, \alpha_{i j} \in \Phi$ such that

$$
v_{i} \eta=\alpha_{i} v_{i}, \quad\left(v_{i}+v_{j}\right) \eta=\alpha_{i j}\left(v_{i}+v_{j}\right)
$$

But since $\eta$ is semilinear, it follows that $\alpha_{i}=\alpha_{j}=\alpha_{i j}$ for all $i$ and $j$. Thus let $\alpha$ be this common scalar. Similarly there exists a scalar $\beta$ such that $v_{i}^{*} \eta=\beta v_{i}^{*}$ for each $i$.

Suppose $i \neq j$; then there exist $\beta_{i j} \in \Phi$ such that

$$
\left(v_{i}^{*}+v_{j}\right) \eta=\beta_{i j}\left(v_{i}^{*}+v_{j}\right)=\beta v_{i}^{*}+\alpha v_{j \vdash}
$$

Thus $\alpha=\beta$.

Let $\gamma \in \Phi$. Then $\left(\gamma v_{i}+v_{j}\right) \eta=\gamma \eta v_{i} \eta+v_{j} \eta=\gamma \eta \alpha v_{i}+\alpha v_{j}$. But there exists $\delta \in \Phi$ such that $\left(\gamma v_{i}+v_{j}\right) \eta=\delta\left(\gamma v_{i}+v_{j}\right)$. Thus $\delta v_{j}=\alpha v_{j}$ or $\delta=\alpha$. Hence $(\gamma \eta) \alpha=\gamma \alpha$ or $\gamma \eta=\gamma$. Thus $\eta$ is a linear mapping.

Let $w$ be any other singular vector in $\Im$. Then there exists $\epsilon \in \Phi$ such that $w \eta=\epsilon w$. Then since $v_{1}, v_{2}, \cdots, v_{k}$ is a basis for a maximal totally singular subspace, there exists $i(1 \leqslant i \leqslant k)$ with $Q\left(v_{i}, w\right) \neq 0$. Then by modifying $w$ by a scalar, we can suppose $Q\left(v_{i}, w\right)=1$. Let $\nu=Q\left(v_{i}^{*}, w\right)$.

$$
Q\left(a v_{i}+b v_{i}^{*}+w\right)=a b Q\left(v_{i}, v_{i}^{*}\right)+a Q\left(v_{i}, w\right)+b Q\left(v_{i}^{*}, w\right)=a b+a+b v .
$$

For $a v_{i}+b v_{i}^{*}+w$ to be a singular vector, we must have 


$$
a=-b v /(b+1)
$$

which clearly has a solution other than $a=b=0$ if $\Phi$ has more than two elements. Thus we make this assumption and suppose $a$ and $b$ satisfy (2).

Then there exists $\mu \in \Phi$ such that $\left(a v_{i}+b v_{i}^{*}+w\right) \eta=\mu\left(a v_{i}+b v_{i}^{*}+w\right)$ since $a v_{i}+b v_{i}^{*}+w$ is singular. But $\left(a v_{i}+b v_{i}^{*}+w\right) \eta=a \alpha v_{i}+b \alpha v_{i}^{*}+\epsilon w$. Thus since $a$ and $b$ are not both zero, we obtain $\mu=\alpha$.

Thus $w \eta=\alpha w$ holds for all singular vectors $w$ if $|\Phi| \geqslant 3$.

If $|\Phi|=2$, then $\alpha=1$ and $\epsilon=1$ since $\eta$ is bijective. Thus again $\epsilon=\alpha$.

Thus $\eta$ is a linear mapping of $\Im$ such that $w \eta=\alpha w$ holds for all singular vectors $w \in \mathcal{Y}$.

Since $\mathcal{Y}$ has a basis consisting of singular vectors, $\eta$ must be scalar multiplication by $\alpha$, proving the lemma.

Thus we have proved the following:

THEOREM 1. Let $\Im=$ Jord $(Q, 1)$ be such that $(Q, 1)$ is a nondegenerate quadratic form with base point of Witt index at least three on a finite-dimensional vector space $\Im$. If $\eta$ is a semilinear bijection of $\Im$ which induces an automorphism of the lattice of inner ideals, then $\eta$ is a semi-isotopy. Moreover, $\eta$ induces the identity automorphism on the lattice if and only if $\eta$ is a scalar multiplication.

3, The algebras $\mathfrak{Q}\left(\mathfrak{D}_{3}, \gamma\right)$ where $\mathfrak{D}$ is an octonion algebra. In our previous paper [7], we used results of Faulkner [2] to show that every automorphism of the lattice of inner ideals of an algebra $\widehat{S}=\mathfrak{Q}\left(\mathfrak{D}_{3}, \gamma\right)$ is induced by a semisimilarity of $\Im$. By a result due to Faulkner [2, Theorem 1.1], the semisimilarities and semi-isotopies of $\Im$ coincide. Thus to prove that any semilinear bijection of $\Im$ that induces an automorphism of the lattice of inner ideals of $\mathcal{Y}$ is a semiisotopy, it suffices to prove that any semilinear bijection of $\Im$ which induces the identity automorphism on the lattice of inner ideals is a semi-isotopy.

By passing to an isotope, we can suppose $\gamma=1$; i.e., the involution in $\mathfrak{D}_{3}$ is the standard involution. We make this assumption from now on.

LEMMA 2. Suppose $\eta$ is a semilinear bijection of $\mathfrak{Y}=\mathfrak{Q}\left(\mathfrak{D}_{3}\right)$ such that $\mathfrak{B} \eta=\mathfrak{B}$ for every inner ideal $\mathfrak{B}$ of $\mathfrak{Y}$. Then $\eta$ is a scalar multiplication.

Proof. $\mathcal{Y}$ has a basis consisting of elements of rank one which we will denote $f_{1}, f_{2}, \cdots, f_{27}$ where each $f_{n}$ is either of the form $e_{i i}(i=1,2,3)$ or $e_{i i}+n\left(a_{k}\right) e_{j j}+a_{k}[i j]$ where $(i j k)$ is a cyclic permutation of $\left(\begin{array}{ll}1 & 2\end{array}\right)$. If $\mathfrak{D}$ is an octonion division algebra $n\left(a_{k}\right) \neq 0$ (since $a_{k} \neq 0$ ). If $\mathfrak{D}$ is a split octonion algebra, then it is clear that we can choose $a_{k}$ such that $n\left(a_{k}\right)=0$ for 
any $a_{k}$ that occurs in our basis (since a split octonion algebra has a basis consisting of noninvertible elements).

Then since $n\left(-a_{k}\right)=n\left(a_{k}\right), n\left(a_{k}\right) e_{i i}+e_{i j}-a_{k}[i j]$ is also an element of rank 1. Let $\delta=n\left(a_{k}\right)$.

Then

$$
\begin{aligned}
& \left(e_{i i}+\delta e_{j j}+a_{k}[i j]\right) \eta=\beta_{1}\left(e_{i i}+\delta e_{j j}+a_{k}[i j]\right) \\
& \left(\delta e_{i i}+e_{j j}-a_{k}[i j]\right) \eta=\beta_{2}\left(\delta e_{i i}+e_{j j}-a_{k}[i j]\right)
\end{aligned}
$$

Thus $\eta$ maps $(\delta+1)\left(e_{i i}+e_{i j}\right)$ to

$$
\left(\beta_{1}+\delta \beta_{2}\right) e_{i i}+\left(\delta \beta_{1}+\beta_{2}\right) e_{j j}+\left(\beta_{1}-\beta_{2}\right) a_{k}[i j] \text {. }
$$

But $e_{i i} \eta=\gamma_{i} e_{i i}$ for some $\gamma_{i} \in \Phi$. Thus $\eta$ maps $(\delta+1)\left(e_{i i}+e_{i j}\right)$ to

$$
\gamma_{i}(\delta+1) \eta e_{i i}+\gamma_{j}(\delta+1) \eta e_{i j}
$$

Then it follows that $\beta_{1}=\beta_{2}$ and

$$
(\delta+1) \beta_{1} e_{i i}+(\delta+1) \beta_{1} e_{j j}=\gamma_{i}(\delta+1) \eta e_{i i}+\gamma_{j}(\delta+1) \eta e_{i j}
$$

Thus

$$
(\delta+1) \beta_{1}=\gamma_{i}(\delta+1) \eta=\gamma_{j}(\delta+1) \eta .
$$

Thus either $\delta+1=0$ or $\gamma_{i}=\gamma_{j}$. If $\Phi$ is a field with more than two elements, by multiplying $a_{k}$ by a scalar, we can force $\delta=n\left(a_{k}\right)$ to be unequal to -1 . If $|\Phi|=2$, then $\mathfrak{D}$ is split, and $n\left(a_{k}\right)=0$ by the choice of $a_{k}$ for the split case. Thus $\gamma_{i}=\gamma_{j}$ for all $i$ and $j$.

The proof of the linearity of $\eta$ is divided into two cases depending on whether $\mathfrak{D}$ is split or not.

Suppose $\mathcal{O}$ is split. Then $\left(e_{i i}+a_{k}[i j]\right) \eta=\beta_{1}\left(e_{i i}+a_{k}[i j]\right)$. Then $\beta_{1}=$ $\boldsymbol{\gamma}_{i}$ (since $\Phi a_{k}[i j]$ is an inner ideal). Thus, for any $a_{k}$ with $n\left(a_{k}\right)=0$, $\left(a_{k}[i j]\right) \eta=\gamma_{i} a_{k}[i j]$. But let $\alpha \in \Phi$; then

$$
\left(\alpha a_{k}[i j]\right) \eta=(\alpha \eta) \gamma_{i} a_{k}[i j] .
$$

But $\left(\alpha a_{k}[i j]\right) \eta=\alpha \gamma_{i} a_{k}[i j]$ (since $n\left(\alpha a_{k}\right)=0$ ). Thus since $\gamma_{i} \neq 0, \alpha=\alpha \eta$ for all $\alpha \in \Phi$. Hence $\eta$ is linear.

Now suppose $\mathfrak{D}$ is an octonion division algebra. Let

$$
b_{1}=e_{i i}+\alpha^{2} \delta e_{j j}+\alpha a_{k}[i j] \text { and } b_{2}=e_{i i}+\delta e_{i j}+a_{k}[i j] \text {. }
$$

Then since $b_{1}$ and $b_{2}$ are elements of rank one, there exist scalars $\beta$ and $\beta^{\prime}$ such that $b_{1} \eta=\beta^{\prime} b_{1}$ and $b_{2} \eta=\beta b_{2}$. Let $\alpha \in \Phi$. Then 


$$
\left(b_{1}-\alpha b_{2}\right) \eta=(1-\alpha) \eta \gamma_{i} e_{i i}+\left(\alpha^{2} \delta-\alpha \delta\right) \eta \gamma_{i} e_{j j}
$$

But

$$
\begin{aligned}
\left(b_{1}-\alpha b_{2}\right) \eta & =b_{1} \eta-(\alpha \eta) b_{2} \eta \\
& =\left(\beta^{\prime}-\alpha \eta \beta\right) e_{i i}+\left(\alpha^{2} \delta \beta^{\prime}-\alpha \eta \beta \delta\right) e_{j j}+\left(\beta^{\prime} \alpha-\alpha \eta \beta\right) a_{k}[i j] .
\end{aligned}
$$

Equating the coefficients of $e_{i i}, e_{j j}$ and $a_{k}[i j]$ in these two equations and performing some simple algebraic manipulations yields $\delta \alpha=(\alpha \eta)(\delta \eta)$ or $(\alpha \delta) \eta=\alpha \delta$. But this holds for all $1 \neq \alpha \in \Phi$ and $0 \neq \delta \in \Phi$. Thus it follows that $\eta$ is linear.

Thus it follows from (3) that $\beta_{1}=\gamma_{i}$. Hence $\eta$ is a linear mapping of $\Im$ such that $b \eta=\gamma_{i} b$ for each $b$ in a basis of 3 . Thus $b \eta=\gamma_{i} b$ for all $b$ in $\Im$, completing the proof of the lemma and the following theorem.

THEOREM 2. Let $\mathfrak{Y}=\$\left(\mathfrak{D}_{3}\right)$ where $\mathfrak{D}$ is an octonion algebra. If $\eta$ is a semilinear bijection of $\Im$ which induces an automorphism of the lattice of inner ideals, then $\eta$ is a semi-isotopy. Moreover $\eta$ induces the identity automorphism on the lattice if and only if $\eta$ is a scalar multiplication.

4. The algebras $\mathfrak{Q}\left(\Delta_{n},{ }^{*}\right)$ where ${ }^{*}$ is a hermitian involution. In this section we prove that if $\eta$ is a semilinear bijection of $\Im=\mathfrak{Q}\left(\Delta_{n},{ }^{*}\right)$, where $\Delta$ is a divison ring, $n \geqslant 3$ and ${ }^{*}$ is a hermitian involution, such that $\mathfrak{B} \eta$ is an inner ideal for every inner ideal $\mathfrak{B}$ of $\mathfrak{Y}$, then $\eta$ is a semi-isotopy of $\mathcal{Y}$. First we prove the following lemma.

Lemma 3. Suppose $\eta$ is a semilinear bijection of $\Im=\$\left(\Delta_{n},{ }^{*}\right)$ such that $\mathfrak{B} \eta=\mathfrak{B}$ for every inner ideal $\mathfrak{B}$ of $\mathfrak{Y}$. Then $\eta$ is a scalar multiplication.

Proof. Since $\Phi e_{i i}=e_{i i} \Im e_{i i}$ is a minimal inner ideal, there exists $\alpha_{i i} \in \Phi$ such that $e_{i i} \eta=\alpha_{i i} e_{i i}$. Similarly since

$$
\left.\Phi\left(e_{i i}+e_{j i}+[i j]\right)=\left(e_{i i}+e_{j i}+[i j]\right)\right\}\left(e_{i i}+e_{j j}+[i j]\right)
$$

(where [ij] denotes $1[i j]$ throughout this paper) is a minimal inner ideal, there exist $\alpha_{i j} \in \Phi$ such that

$$
\left(e_{i i}+e_{i j}+[i j]\right) \eta=\alpha_{i j}\left(e_{i i}+e_{j j}+[i j]\right) .
$$

Since $\Im U_{c[i j]} \subseteq \Phi e_{i i}+\Phi e_{j j}+\Delta[i j]$,

$$
(c[i j]) \eta=\gamma_{i j} e_{i i}+\delta_{i j} e_{j j}+c^{\prime}[i j]
$$

where $\gamma_{i j}, \delta_{i j} \in \Phi, 0 \neq c^{\prime} \in \Delta$ (by regularity of $\Im$ ).

Every matrix in the inner ideal generated by $c[i j]+c[i k]$ has rank at most two over $\Phi$. 
Thus $(c[i j]+c[i k]) \eta$ must have rank two over $\Phi$. But

$$
(c[i j]+c[i k]) \eta=\gamma_{i j} e_{i i}+\delta_{i j} e_{j j}+c^{\prime}[i j]+\gamma_{i k} e_{i i}+\delta_{i k} e_{k k}+c^{\prime \prime}[i k] .
$$

For $(c[i j]+c[i k]) \eta$ to have rank two over $\Phi$, there must exist $\pi, \tau, \epsilon \in$ $\Phi$ (not all zero) such that

$$
\pi\left(\gamma_{i j}+\gamma_{i k}, c^{\prime}, c^{\prime \prime}\right)+\tau\left(\bar{c}^{\prime}, \delta_{i j}, 0\right)+\epsilon\left(\vec{c}^{\prime \prime}, 0, \delta_{i k}\right)=0 .
$$

Thus either $\pi=0$ or $c^{\prime}, c^{\prime \prime} \in \Phi$.

First suppose $c^{\prime}, c^{\prime \prime} \in \Phi$. Let $F=\left(\gamma_{i j}+\gamma_{i k}\right) e_{i i}+c^{\prime}[i j]+c^{\prime \prime}[i k]+\delta_{i j} e_{i j}+$ $\delta_{i k} e_{k k}$. Then

$$
F \Im F=([i j]+[i k]) \Im([i j]+[i k])
$$

since $F \Im F=([i j]+[i k]) \eta \Im([i j]+[i k]) \eta$. If $B=\Sigma \beta_{i} e_{i i}+\Sigma b_{i j}[i j]$ is an arbitrary element of $\Im$, we obtain that $F B F$ has as coefficients of $e_{i j}, e_{k k}$ and $[j k]$, respectively,

$$
\begin{aligned}
& c^{\prime 2} \beta_{i}+c^{\prime} \delta_{i j} t\left(b_{i j}\right)+\delta_{i j}^{2} \beta_{j}, \\
& c^{\prime \prime 2} \beta_{i}+c^{\prime \prime} \delta_{i k} t\left(b_{i k}\right)+\delta_{i k}{ }^{2} \beta_{k}, \\
& c^{\prime} c^{\prime \prime} \beta_{i}+c^{\prime \prime} \delta_{i j} \bar{b}_{i j}+\delta_{i j} \delta_{i k} b_{j k}+\delta_{i k} c^{\prime} b_{i k} .
\end{aligned}
$$

By choosing the $\beta_{i}$ and $b_{i j}$ properly and noting that

$$
F \mathcal{Y} F=([i j]+[i k]) \mathcal{Y}([i j]+[i k])
$$

so that the coefficients of $e_{i j}, e_{k k}$ and $[j k]$ are equal, we obtain $c^{\prime} c^{\prime \prime}=c^{\prime 2}=$ $c^{\prime \prime 2}$ and $\delta_{i j}=\delta_{i k}=0$ (since $c^{\prime}, c^{\prime \prime} \neq 0$ ).

Secondly, suppose $\pi=0$; then an easy inspection shows that $\delta_{i j}=\delta_{i k}=$ 0. By an argument similar to that given in the previous case, we deduce $\vec{c} c^{\prime}=$ $\vec{c}^{\prime} c^{\prime \prime}=\vec{c}^{\prime \prime} c^{\prime \prime}$ which implies that $c^{\prime}=c^{\prime \prime}$.

Thus in all cases, $\delta_{i j}=\delta_{i k}=0$ and $c^{\prime}=c^{\prime \prime}$. Arguing similarly, we deduce $\gamma_{i j}=\gamma_{i k}=0$.

Thus $c[i j] \eta=c^{\prime}[i j]$ and $e_{i i} \eta=\alpha_{i i} e_{i i}$. Since $\left(e_{i i}+e_{j j}+[i j]\right) \eta=\alpha_{i i} e_{i i}+$ $\alpha_{j j} e_{j j}+1^{\prime}[i j]$, we must have $\alpha_{i i}=\alpha_{j j}$ (since $\Phi\left(e_{i i}+e_{i j}+[i j]\right)$ is a one-dimensional inner ideal). Let $\alpha=\alpha_{i i}$.

Let $b \in \Delta$; then

$$
\begin{aligned}
(b[i j] & +1[i k]) \Im(b[i j]+1[i k]) \\
& =\Phi e_{i i}+\Phi\left(n(b) e_{j j}+e_{k k}+\bar{b}[j k]\right)+\Delta\left(1[i j]+b^{-1}[i k]\right) .
\end{aligned}
$$

Suppose $b[i j] \eta=c[i j]$. Then calculating $(c[i j]+\alpha[i k]) \Im(c[i j]+\alpha[i k])$ and 
using the fact that $\eta$ induces the identity map on the lattice of inner ideals, we obtain

$$
n(c) e_{j j}+\alpha^{2} e_{k k}+\alpha \bar{c}[j k]=\beta\left(n(b) e_{j j}+e_{k k}+\bar{b}[j k]\right) \text { for some } \beta \in \Phi .
$$

Thus $\beta=\alpha^{2}$ which implies $\alpha^{2} \bar{b}=\alpha \bar{c}$ or $\bar{c}=\alpha \bar{b}$. Thus $b[i j] \eta=\alpha b[i j]$ for any $b \in \Delta, i$ and $j$. Thus $\eta$ is just the mapping $A \rightarrow \alpha A$ for all $A \in \mathcal{Y}$, proving the lemma.

Before proving a proposition concerning semilinear bijections, which induce automorphisms of the lattice of inner ideals, we will require the following lemma.

Lemma 4. Suppose $\sigma$ is an inner automorphism of $\Delta_{n}$. Then there exists a semi-isotopy $\eta$ of $\mathcal{\Im}=\mathfrak{Q}\left(\Delta_{n},{ }^{*}\right)$ such that $\left(E \Im E^{*}\right) \eta=(E \sigma) \Delta_{n} \cap \Im$ for every idempotent $E \in \Delta_{n}$. Thus any automorphism of the lattice of inner ideals of $\mathcal{J}$ corresponding to an inner automorphism of $\Delta_{n}$ is induced by a semi-isotopy.

Proof. It is well known that the structure group of $\mathcal{Y}$ is the set of all mappings $\tau H_{R}$ where $\tau \in$ Aut $\Delta_{n}$ and there exists $H \in \mathcal{Y}$ such that $\tau^{-1 *} \tau^{*}=$ $I_{H}$ [4]. The group of semi-isotopies consists of all mappings $\sigma H_{R}$ where $\sigma$ is a ring automorphism, not necessarily an algebra automorphism and $H$ is as above. Suppose $\sigma$ is an inner automorphism of $\Delta_{n}$, say $\sigma=I_{A}$; then $\sigma^{-1 *} \sigma^{*}=I_{H_{\sigma}}$ where $H_{\sigma}=A^{-1}\left(A^{-1}\right)^{*}$. Thus $\sigma\left(H_{\sigma}\right)_{R}$ is a semi-isotopy.

Thus

$$
\begin{aligned}
\left(E \Im E^{*}\right) \sigma\left(H_{\sigma}\right)_{R} & =(E \sigma) \Im \sigma\left(E^{*} \sigma\right)\left(H_{\sigma}\right)_{R} \\
& =A^{-1} E A A^{-1} \Im A A^{-1} E^{*} A A^{-1}\left(A^{-1}\right)^{*} \subseteq(E \sigma) \Delta_{n} \cap \Im
\end{aligned}
$$

the desired equality then follows by working with $(E \sigma) \Im(E \sigma)^{*}$ and applying $\sigma^{-1}$, proving the lemma.

We are now ready to prove the following proposition which when combined with Lemma 3 will give us the desired result for this case.

Proposition 2. Suppose $\eta$ is a semilinear bijection of $\Im=\$\left(\Delta_{n},{ }^{*}\right)$, where $\Delta$ is a division ring, $n \geqslant 3$ and * is a hermitian involution, such that $\mathfrak{B} \eta$ is an inner ideal for every inner ideal $\mathfrak{B}$ of $\mathfrak{Y}$. Then $\eta$ is a semi-isotopy.

Proof. By Theorem 3 of [7], there exists a ring automorphism $\phi$ of $\Delta_{n}$ such that $\left(E \zeta^{*}\right) \eta=(E \phi) \Delta_{n} \cap \Im$. Since $\phi$ is a ring automorphism of $\Delta_{n}$, there exists a ring automorphism $\psi$ of $\Delta$ and $C$ invertible in $\Delta_{n}$ such that $\left(a_{i j}\right) \phi=C^{-1}\left(a_{i j} \psi\right) C$. Then let $\eta^{\prime}$ be the semi-isotopy of $\Im$ corresponding to 
the inner automorphism $\phi^{\prime}: A \rightarrow C^{-1} A C$ of $\Delta_{n}$ (Lemma 4). Then consider $\eta\left(\eta^{\prime}\right)^{-1}$. This semilinear bijection induces an automorphism of the lattice of inner ideals which corresponds to the ring automorphism $\left(a_{i j}\right) \rightarrow\left(a_{i j} \psi\right)$ of $\Delta_{n}$. Thus the ring automorphism corresponding to $\eta\left(\eta^{\prime}\right)^{-1}$ fixes all matrix units of $\Delta_{n}$. To show $\eta$ is a semi-isotopy, it suffices to show $\eta\left(\eta^{\prime}\right)^{-1}$ is a semi-isotopy; i.e., it suffices to consider the case in which $\phi$ fixes all $e_{i j}$.

Since everything involved remains unchanged under passage to an isotope, we suppose ${ }^{*}$ is the standard involution.

By a result due to McCrimmon [9, Main Theorem], every inner ideal in $\mathcal{Y}$ is principal. By Proposition 1, $(B \Im B) \eta=(B \eta) \Im(B \eta)$. By our previous results [7, Proposition 4], $(B \mathfrak{\Im} B) \eta=(B \phi) \mathfrak{U} \cap \mathfrak{\Im}$ (where we write $\mathfrak{A}$ for $\Delta_{n}$ to simplify notation). But $(B \eta) \Im(B \eta) \mathscr{A}=(B \eta) \mathscr{A}$ [7, Proposition 4]. Then it follows that $(B \phi) \mathfrak{A}=(B \eta) \mathfrak{A}$ holds for all $B \in \mathfrak{Y}$. Thus $\left(B \eta \phi^{-1}\right) \mathfrak{U}=B \mathfrak{A}$ holds for all $B \in \Im$.

Let $B=e_{i i}$; the only row of $B \eta \phi^{-1}$ that can be nonzero is the $i$ th row. Thus the only row of $B \eta$ that can be nonzero is the $i$ th row. Thus $B \eta \in \Phi B$ for $B=e_{i i}$ (since $e_{i i} \mathfrak{A} \cap \Im=\Phi e_{i i}$ ).

Let $B=[i j]$; then $B \eta \phi^{-1}$ can have only rows $i$ and $j$ unequal to zero; hence the same is true about $B \eta$. Thus $B \eta \in \Delta[i j]+\Phi e_{i i}+\Phi e_{i j}$ and $B \eta \phi^{-1} \in$ $\Phi e_{i i}+\Phi e_{j j}+\Delta e_{i j}+\Delta e_{j i}$.

Thus,

$$
\begin{aligned}
& {[i j] \eta \phi^{-1}=\alpha_{i j} e_{i i}+\alpha_{j i} e_{j j}+b_{i j} e_{i j}+b_{j i} e_{j i}} \\
& {[i k] \eta \phi^{-1}=\alpha_{i k} e_{i i}+\alpha_{k i} e_{k k}+b_{i k} e_{i k}+b_{k i} e_{k i}}
\end{aligned}
$$

Thus,

$$
\begin{aligned}
([i j]+[i k]) \eta \phi^{-1}= & \left(\alpha_{i j}+\alpha_{i k}\right) e_{i i}+\alpha_{j i} e_{j j}+\alpha_{k i} e_{k k} \\
& +b_{i j} e_{i j}+b_{i k} e_{i k}+b_{j i} e_{j i}+b_{k i} e_{k i} .
\end{aligned}
$$

But $[i j]+[i k]$ has row $k=$ row $j$. Thus so does $([i j]+[i k]) \eta \phi^{-1}$. Hence $\alpha_{k i}=\alpha_{j i}=0$. Similarly working with $[i j]$ and $[j k]$ gives $\alpha_{k j}=\alpha_{i j}=0$.

Thus $[i j] \eta \subseteq \Delta[i j]$.

In $e_{i i}+e_{i j}+[i j]$, rows $i$ and $j$ are equal; since this property must be preserved under $\eta \phi^{-1}$ we have $\left(e_{i i}+e_{i j}+[i j]\right) \eta \phi^{-1}=\alpha\left(e_{i i}+e_{i j}+[i j]\right)$ for some $\alpha \in \Phi$ (since $e_{i i} \eta \phi^{-1} \in \Phi e_{i i}$ ).

Suppose $\gamma \in \Phi$; then

$$
\left(e_{i i}+\gamma^{2} e_{i j}+\gamma[i j]\right) \eta \phi^{-1}=\alpha e_{i i}+\alpha\left(\gamma \eta \phi^{-1}\right)^{2} e_{j j}+\alpha\left(\gamma \eta \phi^{-1}\right)[i j] .
$$

We must have row $j$ being $\gamma$ times row $i$. Thus $\alpha\left(\gamma \eta \phi^{-1}\right)=\gamma \alpha$ or $\gamma=\gamma \eta \phi^{-1}$ 
for all $\gamma \in \Phi$. Thus $\eta \phi^{-1}$ is $\Phi$-linear.

Let $c$ be an arbitrary element of $\Delta$; suppose $c[i j] \eta=c_{i j} e_{i j}+\bar{c}_{i j} e_{j i}$. Then

$$
\begin{aligned}
\left(e_{i i}+c[i j]+\right. & \left.n(c) e_{i j}\right) \eta \phi^{-1} \\
& =\alpha e_{i i}+c_{i j} \phi^{-1} e_{i j}+\bar{c}_{i j} \phi^{-1} e_{j i}+n(c) \alpha e_{i j} .
\end{aligned}
$$

But then row $j$ being $\bar{c}$ times row $i$ implies $n(c) \alpha=\bar{c}\left(c_{i j} \phi^{-1}\right)$ or $c \alpha=c_{i j} \phi^{-1}$ or $(\alpha c) \phi=c_{i j}$. Thus $c_{i j}$ does not depend on $i$ and $j$, just on $c$ and $\eta$. Hence we set $c \eta=c_{i j}$.

Then we obtain $\alpha c=c \eta \phi^{-1}$. Thus $\eta=\phi \alpha_{R}$ where $\phi$ is a ring automorphism of $\Delta_{n}$ and $\alpha \in \Phi$.

But it is well known [4] that any such mapping $\eta$ that maps $\Im$ to $\mathcal{Y}$ is a semi-isotopy of $\mathfrak{\Im}$, proving the proposition.

Thus we have proved the following theorem.

THEOREM 3. Let $\Im=\mathfrak{Q}\left(\Delta_{n},{ }^{*}\right)$ where $\Delta$ is a divison ring, $n \geqslant 3$, and * is a hermitian involution. Suppose $\eta$ is a semilinear bijection of $\Im$ which induces an automorphism of the lattice of inner ideals; then $\eta$ is a semi-isotopy. Moreover $\eta$ induces the identity automorphism of the lattice if and only if $\eta$ is a scalar multiplication.

5. The algebras $\mathfrak{Q}\left(\mathfrak{Q}_{n},{ }^{*}\right)$ where $\mathfrak{Q}$ is a split quaternion algebra. In this section, we prove results analogous to those proved in the previous section for the case $\mathfrak{Q}\left(\Delta_{n},{ }^{*}\right)$. The arguments are simplified considerably by the fact that there are "more" inner ideals in this case [9].

Lemma 5. Suppose $\mathfrak{S}=\mathfrak{Q}\left(\mathfrak{Q}_{n},{ }^{*}\right)$ where $\mathfrak{Q}$ is a split quaternion algebra over its center $\Phi$ and $n \geqslant 3$. If $\eta$ is a semilinear bijection of $\}$ such that $\mathfrak{B} \eta=\mathfrak{B}$ for every inner ideal $\mathfrak{B}$ of $\mathfrak{B}$, then $\eta$ is a scalar multiplication.

Proof. Since $\Phi e_{i i}=e_{i i} \Im e_{i i}$ is an inner ideal, there exist $\alpha_{i} \in \Phi$ such that $e_{i i} \eta=\alpha_{i} e_{i i}$. If $a \in \epsilon \mathfrak{Q}$ for some primitive idempotent $\epsilon$ in $\mathfrak{Q}$, then $\Phi a[i j]$ is also an inner ideal; thus there exists $\alpha_{i j a} \in \Phi$ such that $a[i j] \eta=$ $\alpha_{i j a} a[i j]$. McCrimmon [9, Main Theorem] has proved that if $\epsilon$ is a primitive idempotent in $\mathfrak{Q}, \Phi e_{i i}+\epsilon \mathfrak{Q}[i j]$ is a point space (i.e., every $\Phi$ subspace of it is an inner ideal). From this result of McCrimmon's it follows that $\alpha_{i}=\alpha_{i j a}$ for any $i$ and $j$ and for any noninvertible $a \in \mathfrak{Q}$. Let us denote this common value by $\alpha$.

For any $\beta \in \Phi, \Phi\left(\beta e_{i i}+\epsilon[i j]\right)$ is an inner ideal; thus

$$
\left(\beta e_{i i}+\epsilon[i j]\right) \eta=(\beta \eta)\left(e_{i i} \eta\right)+(\epsilon[i j]) \eta=(\beta \eta) \alpha e_{i i}+\alpha \epsilon[i j] .
$$


But $(\beta \eta) \alpha e_{i i}+\alpha \epsilon[i j] \in \Phi\left(\beta e_{i i}+\epsilon[i j]\right)$. Thus $\beta \eta=\beta$ which implies that $\eta$ is linear.

Since $E=\left\{e_{i i} \mid 1 \leqslant i \leqslant n\right\} \cup\{a[i j] \mid a \in \mathfrak{Q}$ noninvertible $\}$ contains a basis for $\mathfrak{Q}\left(Q_{n},{ }^{*}\right)$ and since $\eta$ acts as scalar multiplication on elements of $E$ and is $\Phi$-linear, $\eta$ is a scalar multiplication proving the lemma.

For the algebras $\Im=\mathfrak{Q}\left(Q_{n},{ }^{*}\right)$ there is a result analogous to Lemma 4 . The proof of the result [7] is similar to the proof of Lemma 4 as the determination of the group of semi-isotopies is identical to that of the previous case [4]. We freely use this result in the proof of the following proposition.

Proposition 3. Suppose $\mathfrak{S}=\mathfrak{Q}\left(\mathfrak{\Omega}_{n},{ }^{*}\right)$ where $n \geqslant 3$ and $\mathfrak{Q}$ is a split quaternion algebra over its center $\Phi$. If $\eta$ is a semilinear bijection of $\}$ such that $\mathfrak{B} \eta$ is an inner ideal for every inner ideal $\mathfrak{B}$ of $\mathfrak{Y}$, then $\eta$ is a semiisotopy.

Proof. By [7, Proposition 5], there exists a ring automorphism $\phi$ of $\mathfrak{Q}_{n}$ such that $\left(E \Im E^{*}\right) \eta=(E \phi) \mathscr{U} \cap \mathcal{\Im}$ for all idempotents $E$ of even rank in $\mathfrak{A}$ $\left(=\Omega_{n}\right)$.

Since $\phi$ is a ring automorphism of $\mathfrak{Q}_{n}, \phi$ is given by

$$
\left(q_{i j}\right) \phi=C^{-1}\left(q_{i j} \rho\right) C
$$

for some ring automorphism $\rho$ of $\mathfrak{Q}$ and some $C$ invertible in $\mathfrak{Q}_{n}$.

But then since $\mathfrak{Q}$ is isomorphic to $\Phi_{2}, \rho$ is given by

$$
\left(\alpha_{i j}\right) \rho=B^{-1}\left(\alpha_{i j} \psi\right) B
$$

1. for some automorphism $\psi$ of $\Phi$ and $B$ invertible in $\Phi_{2}$.

Thus $\phi$ is given by

$$
\left(\beta_{i j}\right) \phi=D^{-1}\left(\beta_{i j} \psi\right) D, \quad 1 \leqslant i, j \leqslant 2 n,
$$

where $\psi$ is an automorphism of $\Phi$ and $D$ is invertible in $\Phi_{2 n}$.

Then as in the proof of Proposition 2, we can suppose that $\phi$ stabilizes all the matrix units of $\Phi_{2 n}$; i.e. $\phi$ stabilizes $\epsilon e_{k k}, \epsilon e_{i j}(1 \leqslant i, j, k \leqslant n)$ for $\epsilon=$ $\left(\begin{array}{ll}1 & 0 \\ 0 & 0\end{array}\right),\left(\begin{array}{ll}1 & 1 \\ 0 & 0\end{array}\right),\left(\begin{array}{ll}0 & 0 \\ 0 & 1\end{array}\right)$ and $\left(\begin{array}{ll}0 & 0 \\ 1 & 1\end{array}\right) \in Q$.

Suppose $E$ is an idempotent of rank 2 in $\mathscr{U}$ and consider $E \mathfrak{Y} E^{*}$. Then [7, Lemma 7] $\left(E S^{*}\right) \mathfrak{A}=E \mathfrak{A}=B \mathfrak{A}$ for some $B \in \Im$. Thus $\left(E \Im E^{*}\right) \mathfrak{A}=$ $(B \Im B) \mathfrak{A}$.

Then $(B \Im B) \eta=(B \eta) \Im(B \eta)$ (Proposition 1) which is equal to $(B \phi) \mathscr{U} \cap \Im$.

Thus $\left(\left(B \Im_{B}\right) \eta\right) \mathfrak{U}=(B \eta) \mathfrak{U}=(B \phi) \mathfrak{U}$ which implies that $\left(B \eta \phi^{-1}\right) \mathfrak{U}=B \mathfrak{U}$.

Take $B=\epsilon[i j]$ for the four values of $\epsilon$ described earlier. Then 


$$
B \mathfrak{A}=\epsilon[i j] \mathfrak{A}=\sum_{k} \epsilon \mathfrak{Q} e_{i k}+\sum_{k} \bar{\epsilon} \mathfrak{Q} e_{j k} .
$$

Thus the only possible nonzero rows in $B \eta \phi^{-1}$ are rows $i$ and $j$. The $(i, k)$ th entry of $B \eta \phi^{-1}$ must be in $\epsilon \mathfrak{Q}$ and the $(j, k)$ th entry must be in $\bar{\epsilon} \mathfrak{Q}$. Since $\phi$ maps $\epsilon \mathfrak{Q}$ to $\epsilon \mathfrak{Q}$ and $\overline{\epsilon Q}$ to $\bar{\epsilon} \mathfrak{Q}$ and $\eta$ maps $\mathcal{Y}$ to $\mathcal{Y}$, it follows that the only nonzero entries in $B \eta$ can be in the $(i, j)$ th and $(j, i)$ th positions and these entries must be conjugate under the involution in $\mathfrak{Q}$. From that and the fact that $(B \eta)_{i j} \in \epsilon \mathfrak{Q}$ and $(B \eta)_{j i} \in \bar{\epsilon} \mathfrak{Q}$, it follows that $(B \eta)_{i j} \in \Phi \epsilon$.

Then by an argument similar to that used in the proof of Lemma 5 , it follows that there exists $\alpha \in \Phi$ such that $e_{i i} \eta=\alpha e_{i i}$ and $\epsilon[i j] \eta=\alpha \epsilon[i j]$ for all $i, j$ and $\epsilon$ as described above. From this it follows that $A \eta=\alpha A \psi$ for some automorphism $\psi$ of $\Phi$. By the determination of the group of semi-isotopies [4], it follows that $\eta$ is a semi-isotopy.

Thus we have proved the following theorem.

THEOREM 4. Suppose $\mathcal{J}=\$\left(Q_{n}, *\right)$ where $\mathfrak{Q}$ is a split quaternion algebra over its center $\Phi$ and $n \geqslant 3$. A semilinear bijection $\eta$ of $\mathcal{Y}$ induces an automorphism of the lattice of inner ideals if and only if $\eta$ is a semi-isotopy. Moreover $\eta$ induces the identity automorphism if and only if $\eta$ is a scalar multiplication.

6. The algebras $\mathfrak{A}^{+}$where $\mathfrak{A}$ is simple artinian. In this section we suppose that $\mathfrak{Y}=\mathfrak{U}^{+}$where $\mathfrak{U}$ is a simple artinian algebra; then $\mathscr{U}$ is isomorphic to $\Delta_{n}$ where $\Delta$ is a division ring. Under the further assumption that $n \geqslant 3$ we prove that every semilinear bijection of $\mathfrak{A}^{+}$which induces an automorphism of the lattice of inner ideals is a semi-isotopy. First we prove the following proposition.

Proposition 4. Suppose $\mathfrak{U}=\Delta_{n}$ where $\Delta$ is a division ring and $n \geqslant 3$. If $\eta$ is a semilinear bijection of $\mathfrak{A}$ which induces an automorphism of the lattice of inner ideals, then $\eta$ has necessarily one of the two forms.

$$
X \rightarrow P(X \rho) Q \text { or } X \rightarrow P^{t}(X \epsilon) Q
$$

where $P$ and $Q$ are invertible in $A, \rho$ is an automorphism of $\Delta$ and $\epsilon$ is an anti-automorphism of $\Delta$.

Proof. Let $R$ (resp. $L$ ) denote the lattice of right (left) ideals of $\mathscr{A}$. Then by an argument similar to that used in the proof of Proposition 2 of [7], it follows that either $R \eta=R$ and $L \eta=L$, or $R \eta=L$ and $L \eta=R$.

Suppose $R \eta=R$. Then there exist ring automorphisms $\phi$ and $\psi$ of $\mathscr{A}$ such that $\eta \mid R=\phi$ and $\eta \mid L=\psi$. Then there exist ring automorphisms $\sigma$ and $\tau$ of $\Delta$ and invertible elements $P$ and $Q$ in $\mathscr{A}$ such that $X \phi=P^{-1}(X \sigma) P$ and $X \psi=Q^{-1}(X \tau) Q$. 
Then consider the mapping $\eta^{\prime}: X \rightarrow P(X \eta) Q^{-1}$. It follows that for any $E, F \in \mathscr{U}$,

$$
(E \mathscr{U}) \eta^{\prime}=(E \mathscr{Q}) \sigma P Q^{-1} \text { and }(\mathscr{U} F) \eta^{\prime}=P Q^{-1}(\mathscr{U} F) \tau
$$

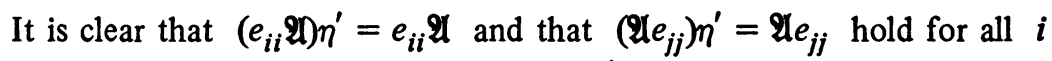
and $j$. But $\Delta e_{i j}=e_{i i} \mathfrak{\mathscr { A }} \cap \mathfrak{\mathscr { A }} e_{i j}$. Thus $\left(\Delta e_{i j}\right) \eta^{\prime}=\Delta e_{i j}$. Thus there exist $d_{i j} \in \Delta$ such that $e_{i j} \eta^{\prime}=d_{i j} e_{i j}$.

It is also clear that $\eta^{\prime}$ fixes the minimal left ideal that has columns $j$ and $k$ equal and all other columns equal to zero. Thus $\eta^{\prime}$ fixes $\Delta\left(e_{i j}+e_{i k}\right)$ from which it follows that $d_{i j}=d_{i k}$. Similarly one obtains $d_{k j}=d_{i j}$. Thus all the $d_{i j}$ are equal. Let $d$ be this common value.

Let $c \in \Delta$, then $\left(e_{i j}+c e_{k j}\right) \eta^{\prime}=d e_{i j}+c^{\prime} e_{k j}$. Since $\left(c e_{i j}+c e_{k j}\right) \eta^{\prime}$ must have rows $i$ and $k$ equal, $c^{\prime}$ depends only on $c$ and $\eta$ and is independent of $k$ and $j$. Define a mapping $\phi$ of $\Delta$ to itself by $c \phi=c^{\prime} d^{-1}$. Then since $c \rightarrow c^{\prime}$ is a bijection of $\Delta$, so is $\phi$.

Next we want to show that $\phi$ is an automorphism of $\Delta$. Let $b, c \in \Delta$; then

$$
\begin{aligned}
& \left(b e_{i j}+c e_{i j}\right) \eta^{\prime}=\left((b+c) e_{i j}\right) \eta^{\prime}=(b+c) \phi d e_{i j} \\
& \left(b e_{i j}+c e_{i j}\right) \eta^{\prime}=\left(b e_{i j}\right) \eta^{\prime}+\left(c e_{i j}\right) \eta^{\prime}=(b \phi+c \phi) d e_{i j}
\end{aligned}
$$

Thus $(b+c) \phi=b \phi+c \phi$. To show that $\phi$ preserves multiplication, let $b, c \in \Delta$. Then

$$
\begin{aligned}
\left(e_{i j}+b e_{k j}\right) c \mathfrak{U} & =\left(e_{i j}+b e_{k j}\right) \mathfrak{U} ; \\
\left(e_{i j}+b e_{k j}\right) c & =c e_{i j}+b c e_{k j} .
\end{aligned}
$$

Thus $\left(\left(e_{i j}+b e_{k j}\right) c\right) \eta^{\prime}=(c \phi) d e_{i j}+(b c) \phi d e_{k j}$. But $\left(e_{i j}+b e_{k j}\right) \eta^{\prime} \mathscr{U}$ is the right ideal with row $k$ equal to $b \phi$ times row $i$. Thus $(b \phi)(c \phi) d=(b c) \phi d$, or since $d \neq 0,(b \phi)(c \phi)=(b c) \phi$ as required.

Thus $X \eta^{\prime}=(X \phi) d$ where $\phi$ is a ring automorphism of $\Delta$ and $d \in \Delta$.

Thus

$$
P X \eta Q^{-1}=(X \phi) d \quad \text { or } \quad X \eta=P^{-1}(X \phi) d Q=P^{-1}(X \phi) Q^{\prime}
$$

where $\phi \in$ Aut $\Delta$.

Finally, we must consider the case in which $R \eta=L$. Then there exists an anti-automorphism $\zeta$ of $\mathscr{A}$. Then $R \eta \zeta^{-1}=R$. Thus by the previous case $X \eta \zeta^{-1}=P^{-1} X \phi Q$ or $X \eta=Q \zeta X \phi \zeta\left(P^{-1}\right)$. But since $\phi \zeta$ is an anti-automorphism of $\mathfrak{A}$, it is given by $X \phi \zeta=C^{-1 t}(X \epsilon) C$ where $\epsilon$ is an anti-automorphism of $\Delta$. Thus $X \eta=A^{t}(X \epsilon) B$ where $\epsilon$ is an anti-automorphism of $\Delta$ and $A, B \in$ $\Delta_{n}$ are invertible, proving the proposition. 
It is well known [4] that the mappings which appear in the conclusion of the proposition are precisely the semi-isotopies of $\Delta_{n}$. Thus we have the following corollary.

COROLlary. Suppose $\eta$ is a semilinear bijection of $\Delta_{n}^{+}$which induces an automorphism of the lattice of inner ideals; then $\eta$ is a semi-isotopy.

It remains to determine which semilinear bijections induce the identity automorphism of the lattice of inner ideals. The result in this case is the following lemma.

LEMma 6. Suppose $\Im=\Delta_{n}^{+}$where $\Delta$ is a division ring and $n \geqslant 3$. If $\eta$ is a semilinear bijection of $\mathfrak{Y}$ such that $\mathfrak{B} \eta=\mathfrak{B}$ for all inner ideals $\mathfrak{B}$ of $\Im$, then $\eta$ is a scalar multiplication by an element of the center of $\Delta_{n}(=$ center of $\Delta$ ).

Proof. Since $\eta$ induces the identity automorphism on the lattice, we must have $R \eta=R$. As in the proof of the previous proposition, there exists $d \in \Delta$ such that $e_{i j} \eta=d e_{i j}$ for all $i$ and $j$. Suppose $c \in \Delta$; then $\left(e_{i j}+c e_{k j}\right) \eta=d e_{i j}+(c \phi) d e_{k j}$. But since row $k$ must be $c$ times row $i, c \phi=c$. Thus $\phi$ is the identity map on $\Delta$. Thus $X \eta=X d$ for all $X \in \Delta_{n}$. Consider $\mathscr{U}\left(e_{i j}+c e_{i k}\right)$. This left ideal has column $k$ equal to $c$ times column $j$. $\left(\mathscr{A}\left(e_{i j}+c e_{i k}\right)\right) \eta$ has column $k$ equal to column $j$ times $d^{-1} c d$. Thus we must have $d^{-1} c d=c$ for all $c \in \Delta$ or $d \in$ center $\Delta$.

Thus $X \eta=\mu X$ for some $\mu$ in the center of $\Delta$ as required to prove the lemma.

Thus we have proved the following theorem.

THEOREM 5. Let $\Im=\Delta_{n}^{+}$be such that $\Delta$ is a division ring and $n \geqslant 3$. If $\eta$ is a semilinear bijection of $\}$ which induces an automorphism of the lattice of inner ideals, then $\eta$ is a semi-isotopy. Moreover, $\eta$ induces the identity automorphism on the lattice if and only if $\eta$ is a scalar multiplication.

\section{REFERENCES}

1. J. Dieudonné, La géométrie des groupes classiques, 3ième éd., Ergebnisse der Mathematik und ihrer Grenzgebiete, Band 5, Springer-Verlag, Berlin and New York, 1971. MR 46 \#9186.

2. J. Faulkner, Octonion planes defined by quadratic Jordan algebras, Mem. Amer. Math. Soc. No. 104 (1970). MR 42 \#6063.

3. N. Jacobson, Lectures on quadratic Jordan algebras, Tata Institute of Fundamental Research, Bombay, 1969.

4. - Some groups and Lie algebras defined by quadratic Jordan algebras, Bull. Amer. Math. Soc. (to appear).

5. N. Jacobson and K. McCrimmon, Quadratic Jordan algebras of quadratic forms with base points, J. Indian Math. Soc. 35 (1971), 1-45. 
6. J. M. Katz, Automorphisms of the lattice of inner ideals of certain quadratic Jordan algebras, Doctoral Dissertation, Yale University, New Haven, Conn., 1972.

7. - Isomorphisms of the lattice of inner ideals of certain quadratic Jordan algebras, Trans. Amer. Math. Soc. 185 (1973), 309-329.

8. K. McCrimmon, A general theory of Jordan rings, Proc. Nat. Acad. Sci. U.S.A. 56 (1966), 1072-1079. MR 34 \#2643.

9. - Inner ideals in quadratic Jordan algebras, Trans. Amer. Math. Soc. 159 (1971), 445-468. MR 43 \#4871.

DEPARTMENT OF MATHEMATICS, UNIVERSITY OF MISSOURI-ST. LOUIS, ST. LOUIS, MISSOURI 63121 